\title{
Carnets
}

Revue électronique d'études françaises de l'APEF

Première Série - 2 Numéro Spécial 10-11 | 2011

D'un Nobel l'autre

\section{Textes brefs de Le Clézio: incertitudes et lueurs}

\section{Isabelle Roussel-Gillet}

\section{OpenEdition}

\section{Journals}

Édition électronique

URL : http://journals.openedition.org/carnets/5479

DOI : 10.4000/carnets.5479

ISSN : 1646-7698

Éditeur

APEF

Édition imprimée

Date de publication : 1 janvier 2011

Pagination : 67-80

Référence électronique

Isabelle Roussel-Gillet, «Textes brefs de Le Clézio: incertitudes et lueurs », Carnets [En ligne], Première Série - 2 Numéro Spécial 10-11 | 2011, mis en ligne le 16 juin 2018, consulté le 19 avril 2019. URL: http://journals.openedition.org/carnets/5479; DOI : 10.4000/carnets.5479

Carnets est mis à disposition selon les termes de la licence Creative Commons - Atribution - Pas d'utilisation commerciale 4.0 International. 


\title{
TEXTES BREFS DE LE CLÉZIO: INCERTITUDES ET LUEURS
}

ISABELLE ROUSSEL-GILLET

Université de Lille 2

Isabelle.roussel@univ-lille2.fr

\begin{abstract}
Résumé
Chez Le Clézio, les figures d'entre-deux sont des opérateurs de paradoxes faisant de la littérature un lieu d'incertitudes et le tressage de motifs contribue à une esthétique paradoxale de l'éclat et du lien. Cette esthétique repose aussi sur une éthique du dénuement, un "régime du rien" que nous analysons dans des textes brefs des années 1973, 1978-1980 et 2001 (Mydriase, Trois villes saintes, La Montagne du dieu vivant et L'Enfant de sous le pont) afin de démontrer comment ces textes se lisent à l'aune de la déconstruction, des théories du chaos. Entre nuit noire et aveuglante lumière, Le Clézio privilégie les éclats, les lueurs et les survivances ou revivances.
\end{abstract}

\begin{abstract}
In Le Clézio, the figures of in-between-two are paradoxes operating to make litterature a space of doubt. The weaving of patterns contributes to a paradoxal aesthetics of sparkle and link. This aesthetics is also based on an ethics of bareness, an election of nothing, which we analyze in short stories written in 1973, 1978-1980 and 2001 (Mydriase, Three holly towns, The God'alive Montain et The Child under the bridge) to show how these texts are read from the standpoint of deconstruction and chaos theory. In between black night and blinding light, Le Clézio favours sparkles, gleams, and survivals or revivals.
\end{abstract}

Mots-clés: nouvelle, conte, minimalisme, chaos, incertitude, lumière

Keywords: story, tale, minimalism, chaos, doubt, light 
Le Clézio fait figure d'inclassable, souvent isolé dans les anthologies comme écrivain de l'ailleurs. Or il est possible de l'inscrire parmi ses contemporains dans l'histoire littéraire si l'on prend en considération trois tendances: l'écriture de soi (et son jeu de levée de masques d'Onitsha à L'Africain)), l'écrit de restitution (le rapport à l'Histoire) et le roman polyphonique. Cet écrivain, si "reconnu" quand on liste ses prix outre le Nobel, ne se situe pas dans un parcours à la Proust ou à la Balzac dans l'obsession de l'CEuvre. Bien qu'écrivant "toujours le même livre", il s'est plutôt engagé tel Valéry ou Benjamin comme passant, dans un éclatement particulièrement fertile. Ce sont ces éclats, cet étoilement thématisé dans l'œuvre dont chaque livre explore un possible qui nous retiennent. La critique en a fait la métaphore du désenchantement, dans la séduction du préfixe dé au travail, autant dans le démontage critique que dans le corps littéraire, la déconstruction s'inscrivant dans le versant intellectuel de la modernité, de Derrida. II est vrai que Le Clézio, selon une pensée moderne, privilégie le réseau à l'idée de totalité. Son écriture en offre diverses déclinaisons: par fragments hétérogènes et nomadisme (Le Livre des fuites), par archipels thématisés (La Quarantaine), par infinitudes des espaces lisses, au sens deleuzien (Désert). Cette option participe d'une esthétique de la rupture, si bien nommée par l'académie du prix Nobel. Toutefois les fables même brisées n'en sont pas moins des fables (Pawana). Le Clézio n'ébranle pas comme Rimbaud, il privilégie plutôt un art du flottement (qui sait manier la chronotopie, les ambivalences et les modalisateurs) et fait de la littérature "un lieu d'incertitude"1. Le mouvement est de fait dans sa trame romanesque toujours double: entre désoeuvrement (processus au sein du texte) et ouvrage (comme Pénélope), entre "je" et "l'autre", entre les niveaux narratifs d'un roman à deux histoires (roman double) dont l'une prend le statut de légende, comme c'est le cas pour Désert, Onitsha et La Quarantaine. C'est ce double mouvement qui, entre autres, permet les écarts et les entre-deux. Dans cet espace tiers du jeu, se noue progressivement une poétique du lien. Le Clézio a baptisé "forêt de paradoxes" son espace littéraire des entre-deux. Dans la forêt amérindienne de lianes on se perd, sauf à suivre le fleuve tout tracé, alors acceptons de nous perdre dans des entre-deux, dans l'incertain, qui est, selon Gruzinski, l'ordre du métissage.

Le tressage dans les récits de détails participe de la construction double, ne serait-ce que par le changement d'échelle qu'elle induit (et qui est en même temps potentiellement nié, nous le verrons puisque la position éthique leclézienne est de refuser les échelles, le détail au sens deleuzien n'étant qui plus est pas la partie d'un tout). Le terme tressage, repris de Ricardou, désigne les liens rhizomatiques établis, les "pontages", les "résonances"². Tout comme la polyphonie, le tressage travaille le narratif en orientant une lecture plurivectorielle.

\footnotetext{
1 "Un lieu d'incertitude", entretien avec C. Dupont-Monod, Page des librairies, novembre 2000, p. 8.

2 Nombreux sont les vocables utilisés par la critique de l'œuvre leclézienne: échos, motifs, résonances, constellations.
} 
À la différence de la polyphonie qui repose sur les voix (et voies narratives), le tressage relève d'une structuration de motifs, qui font retour et marquent ainsi l'espace et le temps.

Nous proposons d'analyser ce régime des motifs tissés dans le dénuement qui construisent la littérature comme lieu d'incertitude à travers des textes brefs, peu prisés par la critique, du fait peut-être de leur nature qui ne relèvent ni d'une avant-garde formaliste (le puzzle morcelé du Procès-verbal), ni du roman polyphonique (Révolutions): Trois villes Saintes et Mydriase appartiennent à la veine poétique de l'œuvre, à sa part magique, incantée et L'Enfant de sous le pont est un récit dédié aux enfants, paru dans un circuit de diffusion peu médiatisé, celui de l'aide à la lecture dans les milieux défavorisés. La nouvelle "La Montagne du dieu vivant" propose une attention au presque rien, relayée par les illustrations de Georges Lemoine, dans un régime du minimal que préfigurent des passages romanesques comme la description d'un galet dans Les Géants. Ces récits, où Le Clézio ne raconte "presque rien" ou "presque quelque chose", laissent paradoxalement la trace d'un essentiel. Si des critiques ${ }^{3}$ ont déjà présenté Le Clézio comme écrivain "engagé" notamment par sa dénonciation des exploitations et dominations, notre approche interroge quant à elle le mode mineur de l'interrogation "engageante" 4 par ce choix éthique que constitue un certain régime du rien, du petit, du détail, du numineux fugitif.

Nous suivrons l'ordre chronologique (1973- 1978/1980- 2001) afin de démontrer l'inclinaison de l'oeuvre de la nuit noire aux lueurs, c'est dire du désenchantement postmoderne à la survivance (et non à l'espoir béat ou aveuglé) ${ }^{5}$.

\section{Désenchantement et nuit noire?}

Brouillage des données spatiales comme lorsque Naja Naja flotte dans les étoiles, changement d'échelle, déterritorialisation thématisée, style vibratoire (de flux et d'ondes) et répétitif, fragmentation du texte soumis à la confusion mentale du personnage narrateur Adam Pollo contribuent à une esthétique de l'éclatement. L'œuvre des années 70 met en scène des procédés empruntés aux sciences du chaos, qui définissent aussi l'esthétique postmoderne: le brouillage des genres (Mydriase essai ou souffle poétique?), la déconstruction du narratif, la non-linéarité de l'intrigue, les collages. Mais passons les tous premiers livres de fuites pour lire Mydriase (1973). Écrit au mode impersonnel, ce récit peut se résumer ainsi: dans la nuit noire, "sans même une luciole" (9) que les mots ont désertée, "on" est pétrifié, immobile jusqu'à devenir cette nuit de froid et de glace. La dilatation des

\footnotetext{
${ }^{3}$ Thèse de 2004 de Marina Salles publiée en deux tomes $(2006,2007)$, puis travaux de Claude Cavallero (2008, 2009).

${ }^{4}$ Nous pensons que l'œuvre engage l'empathie et diffère de l'engagement sartrien. L'ère du soupçon a remis en question les effets de l'engagement.

${ }^{5}$ Didi-Huberman, Survivance des lucioles, Minuit, 2009.
} 
yeux dans le noir fait voir progressivement des faces cassées, une fêlure, une lettre, un dessin de fœtus, de la "poudre de mica". L'événement est la venue du regard, de la possibilité de voir, de forer, dans un balbutiement du langage. L'être tendu dans le désir de voir fait alors advenir le regardé et ce qu'on voit nous regarde, expérience phénoménologique suscitée par la mydriase, c'est-à-dire la dilatation des yeux provoquée par le "breuvage noir" concocté à base de feuilles de datura.

L'instabilité des pronoms contribue aisément au flottement, comme l'atteste cette suite de phrases: "on est à l'intérieur de sa tête", "devant les yeux d'un géant" "ou bien peutêtre qu'on est soi-même ce géant" "dans l'espèce de baignoire où vous devenez de plus en plus petit"... La nuit noire est opposée à la lumière dure à l'électricité, et à la peur, ce qui métonymiquement désigne la société occidentale. La sécurité aveuglante du soleil (52) est l'œil panoptique de la ville postmoderne qui dérègle l'écriture en ressassement du mot REGARDAIT qui crève la page par ses majuscules. II faut échapper à la prédation de la conscience pour passer au côté du regardé, des oiseaux, de la nescience, de son corps, ce qui peut se produire lors de la prise de datura ${ }^{6}$. La finalité est de rompre "en miettes la connaissance du langage".

“Devant les yeux riens", un rien repris (24) et décliné puisqu' "on n'a rien à dire. II n'y a pas d'histoire". (36) puis cette phrase isolée par deux blancs "lls vont apparaître maintenant”. Qui va apparaître? Les parties du corps démembrés, les sons, les mots? Le regard s'affole en quête de "nébuleuses, novae, quasars" (26) et les majuscules annoncent CELA A COMMENCÉ. Une piste interprétative nous est donnée: "On est là, entre le rien et le presque quelque chose". La parturition annoncée par le dessin de Velickovik au frontispice est une "terreur de la lumière". Dès lors c'est le regard, source de lumière, qui fabrique ce qui brûle, le désert, le sable et l'énonciation s'accélère. "Poussières d'étoiles", "étincelles éclatantes", "étoiles de lumière", "minuscules soleils", les yeux boivent toutes ces lueurs et des noms d'étoiles sont semés en voûte sur les pages 45 et 46 . L'isotopie est bien celle de l'éclatement mais aussi celle de l'éclat lumineux, de la lumière d'un œil-univers infini. La quête de lumière signale une sortie du désenchantement, elle est aussi le signe d'un entredeux inspiré par la pensée primitive: le moment où l'on passe du côté des esprits, de "l'autre côté", le moment de naître aussi. À la lecture de ce texte, soit on l'interprète au regard de l'extase chamanique par comparaison avec le texte "Le Génie du datura" qui relate précisément la consommation de datura, la nuit noire est alors rituellement le moment propice pour boire l'Iwa des indiens Emberas, soit on l'interprète en suivant les associations

\footnotetext{
6 "Le Génie du datura", 15 janvier 1973, n919, p. 97. Un autre extrait est publié Le Jardin des serpents, n918, 15 avril 1973, pp. 52-63. Le Clézio ayant dit du Génie du datura qu'il est le seul livre qu'il eut voulu ne pas avoir publié, alors qu'il n'en a publié que deux extraits, a sans doute aussi refroidi certains critiques, pour respecter les scrupules de l'écrivain de trahir la confiance de ceux qui l'ont initié, de n'être pas compris par les Occidentaux qui n'ont pas la même conception de la prise d'hallucinogènes.
} 
de "l'invasion imaginaire" ${ }^{7}$ faite de contradictions qui le gagne, soit on laisse les dessins de vulves et de bouches béantes de Vélikovitck qui suggèrent cri et trauma prendre le pas, soit, encore, on l'interprète à l'aune d'une métaphore qui pense la nuit noire comme mort et la lumière crue comme espace aveuglant des sociétés de contrôle, alors, entre ces deux excès du noir et de l'aveuglant, les lueurs figurent une possible survivance. C'est ici qu'il nous faut commencer à discuter de la place de Le Clézio: œuvre crépusculaire du désenchantement à ses débuts, jamais l'œuvre n'est illuminée du soleil de la connaissance. Rappelons-nous que le prophète Adam Pollo, "dans la lumière du plein midi", comme l'écrit Michel Foucault, est au final tenu enfermé "pour un temps indéfini et immobile dans la chambre quadrillée d'un asile". "Refermé sur lui-même, le temps se répartit maintenant sur cet échiquier de barreaux et de soleil. Grillage qui est peut-être la grille du langage". "Point aveugle, impensable de la littérature" nous dit encore Foucault ${ }^{8}$. C'est bien à cette tache aveugle ${ }^{9}$ que s'éprouve l'écriture de Mydriase: l'œil "voit dans tous les temps et tous les lieux, tache aveugle qui avance dans le ciel nocturne" (M, 62).

La lecture du "Génie du Datura" nous en convainc: Iwa, reine de la nuit magique, donne "le repos de la lumière douce, fraîche, bleue" et non la "torpeur" du jour, le "Soleil, hypnotique, impitoyable et meurtrier" (GD, 101). ${ }^{10}$ Au réveil, le narrateur indique en note de bas de page ne pas reconnaître d'emblée le pont qu'il a vu, celui du Panama qui relit les Amériques du Nord et du Sud, un pont qu'il décrit comme une "constellation d'étoiles".

"Sur le sol noir les choses brillent avec un éclat forcené, dans le genre d'étoiles, lucioles, yeux phosphorescents, lampes, pièces d'or. (Est-ce là l'origine de la légende d'Iwa montreuse d'or?)" (GD, 104)

La vision suivante est celle d'une toile d'araignée, d'un labyrinthe derrière lequel le narrateur aperçoit le paysage brisé, en morceaux qui "ne s'ajustent pas" (GD, 109). Puis le passage de l'autre côté lui fait voir "la similarité des fleuves et du sang", des objets naturels pensés par la théorie des fractales, nous y reviendrons.

La lecture de Mydriase oblige le lecteur à lâcher une posture, une attente quant à "quelque chose qui doit se passer", à chercher une intrigue, un sens car "l'histoire n'est pas le sens. Le sens, c'est voir"11, à la condition d'une lumière, dont nous suivons les éclats, au double sens du terme de morceau et de lumière d'un livre à l'autre.

\footnotetext{
${ }^{7}$ Gérard de Cortanze, entretien dans le Magazine littéraire, N362, p. 34.

${ }^{8}$ Michel Foucault, "Le langage de l'espace", Critique, éditions de Minuit, 1946, pp. 378-382.

${ }^{9}$ En optique, la tache aveugle désigne un espace invu du fait des cônes et des bâtonnets. II n'est pas anodin que Mydriase soit publié chez Fata Morgana, qui désigne un phénomène optique de mirages.

${ }^{10}$ Le dieu du Soleil Saquasohuh est celui de la guerre annoncée dans "Peuple du ciel", l'apocalypse promise, en écho à celle du Vietnam en filigrane du récit historicisé par la présence d'un soldat, le soleil est celui des Icares des temps modernes qui se brûlent à l'électricité.

${ }^{11}$ Bernard Dewulf, Peintures et scénarios, Robert Devrient, 2009, pages 28 et 29.
} 


\section{L'entre-deux comme espace de l'incertitude, opérateur de paradoxes}

Trois villes saintes, écrit entre 1971 et 1973, est publié en 1980, la même année que Désert et évoque les Séparés, un groupe maya qui refuse l'intégration. Composé de trois textes $^{12}$ dont chacun porte le nom d'une ville sacrée Chancah, Tixcacal, Chun Pom, le recueil raconte, par le on impersonnel, l'exode d'un groupe d'hommes sur une route encadrée d'arbres "maigres, momifiés", aux "feuilles couvertes de poussière" (10). Le paysage est exclusivement empli de "chaque parcelle couleur de cendre", de "cette poudre immatérielle qui ralentit tout", le récit y compris qui ne démarre pas. Le livre ne relate en effet qu'une seule action: la marche dans un style anaphorique en ritournelles et volutes qui suspendent le temps et déterritorialisent. Avec Trois villes saintes, le dénuement, fondé sur la redite, contribue à une forme de déréalisation, de flottement des repères. Tout concourt à annuler le récit. D'ailleurs l'évocation de la poussière est explicitement associée au gel des mots: "c'est comme si les paroles n'avaient jamais existé". Et la marche du "on avance", est contrariée par le "ils sont immobiles" de la même phrase, ou par la précision ultérieure: "on avance comme à reculons". C'est dire que dès l'incipit le mouvement est double, paradoxal, mais non neutralisé pour autant, métaphore du mouvement infini de la création littéraire. Opérateur de glissement, l'adjectif "blanc" affecté aux hommes puis aux soldats du passé crée une confusion des temps qui permet d'introduire un extrait du Chilam Balam ${ }^{13}$. La thématique de la prolifération des racines qui cherchent l'eau renforce cette écriture de l'envahissement. La dérive poétique emporte le récit, que déréalisent l'expression "comme si", autant que la thématique de la trace, de son évanescence: "on suit les traces, on reconnaît l'itinéraire du rêve", de quoi gommer tout référentiel. Voici tressés ensemble la légende et le poème-rêve leclézien en un corps-texte innervé de liens. La présence du texte mythique se fait par enchâssement avec retrait de marge et contamination de la litanie. Le tarissement de la terre est celui de nos fables: "peut-être qu'on trouvera un jour l'eau, l'eau ancienne, le langage." C'est que l'Histoire est aujourd'hui celle de camions blindés qui passent, hier celle de la conquête. Face à cette réalité, le mythe opère une reconstruction du lien dans cet espace de "mots dérivant comme des îles, muets, muets" (21). La forêt de Chancah "attend". Une simple métaphore "la mer des arbres étroits, brûlés, souffrants" allie

\footnotetext{
12 Le Clézio explique dans une lettre à Georges Lavaudant: "Pour Trois Villes saintes, j'avais pensé à un récit à trois voix, l'une (la mienne) du présent, l'autre du rêve (les paroles de ChilamBalam), la dernière celle de Juan de la Cruz Ceh, le chef insurgé des separados de Chan Santa Cruz, dont les mots sont rapportés par Villas (le livre est à la bibl. du Musée de l'Homme de Paris)“, Archipel Lavaudant, collectif dir. Yan Ciret, Christian Bourgeois éditeur, 1997, pp. 107-108.

${ }^{13}$ Sur le plan historique, les Séparés gardent leurs traditions et rejettent l'alternative d'intégrer au plus bas niveau le mode de vie hispanique. En 1910, contre l'armée gouvernementale, les habitants s'étaient constitués en gardes pour surveiller les lieus saints des Separados, "à Tixcacal Guardia, à Chun Pom, à Chancah de la Vera Cruz".
} 
l'eau et le feu. Le récit se conclut sur notre terre de paradoxes: la société où "dans les tuyaux court l'eau facile" et la forêt de "l'eau morte". La phrase finale offre une chute salvatrice: "Mais c'est du ciel que vient l'eau vraie, un jour". Mais aussitôt après ce verbe au présent de généralité, un futur suffit à maintenir le doute, l'incertitude que nous évoquions en préambule.

Tout comme Mydriase, écrit sous l'effet de la drogue, qui forme un diptyque avec Le Génie du Datura ${ }^{14}$, le passage à une vie éclairée, qui voit au-delà des arbres est servie par la métaphore de la naissance "comme si on naissait à nouveau" et la quête de l'origine "pour trouver le lieu de la naissance". La possibilité même de nouer le récit est défaite en une phrase: "Mais ce sont des rêves n'est-ce pas il ne s'est rien passé". Le style n'a rien de minimaliste, nous sommes loin d'une écriture blanche. L'économie est ailleurs: dans la répétition, la modulation d'une seule action, celle de marcher, et dans le tressage de son paradoxe: le blocage du mouvement. Puisque tout est attente, de l'eau, de la vengeance, des mots. Le lecteur est entre deux questions: "qu'est-ce qui s'est passé" (la nouvelle selon Deleuze ${ }^{15}$ ou l'ab origine) et "qu'est-ce qui va se passer?" (le conte ou l'in fine). Et c'est cet entre-deux genres dont Le Clézio fait le lieu du texte. L'appel du sens est entre deux temps, dans ce flottement qui rejette à l'horizon la promesse d'un événement-avénement. L'écriture de la suspension connaît un envol de quatre pages écrites sans ponctuation, à l'imparfait, qui réalise la prophétie, où l'eau répondait aux prières. Les temps grammaticaux jouent donc leur rôle pour creuser l'écart temporel. Dans le second texte Tixcacal arrive la nuit, le gel du présent sur fond de destruction: "les rêves sont interrompus, il n'y a pas de souvenirs" (41). Les Séparés refusent le monde des villes et de la prédation, Le Clézio en fait des figures de résistance, voire de rédemption, car c'est avec eux qu'est la lumière. Construites sur la tension du regard et sur la tenue du corps, ces pages se ferment à nouveau sur le nonévénement, ainsi encore reculé: "il ne s'est rien passé, rien en vie". Seulement la nuit est passée, le soleil se lève, et les femmes marchent vers le puits. Enfin, l'ultime partie Chun Pom, s'ouvre sur des hommes accablés par la sécheresse, assis autour de l'arbre ceiba et tombent enfin "les premières gouttes d'eau froide". La légende est une force non un fardeau, ainsi de la fonction de la littérature exprimée par Annah Harendt. Nous l'avons vu: la défabulation au niveau de la phrase, du récit à reculons ne suffit pas à nier la fable tissée dans la légende, celle de la pluie venant.

\footnotetext{
${ }^{14}$ Texte bref paru aux Cahiers du chemin.

${ }^{15}$ Mille plateaux, p. 235. Bruno Thibault et Claude Cavallero posent cette distinction dans leur introduction au numéro 2 des Cahiers JMG Le Clézio, Contes, nouvelles et romances, Paris, éditions Complicités, 2009. Ni Mydriase et Trois villes saintes ne se laissent pas enfermés dans les catégories génériques (conte, nouvelle, romance) et ne figurent pas dans le corpus de l'ouvrage.
} 
Comme nous le constatons Le Clézio ne verse pas dans un minimalisme positif, béat puisque le récit est travaillé de l'intérieur par une fragilité, un désenchantement ${ }^{16}$. La plénitude mystique parfois entrevue est toujours écrite en contrepoint d'une scène douloureuse (pour notre corpus: la conquista, la parturition traumatique pour le nouveau né, la solitude, la précarité). Le minimalisme, au sens de dénuement, d'un "minimalisme de l'esprit", selon l'expression de l'auteur, tient pour une part à une fascination pour la disparition (d'un peuple, d'un signe), d'où le goût avéré pour la trace, comme figure paradoxale.

\section{Éthique du dénuement: une pierre et un galet}

Les positions critiques de l'auteur par rapport à la culture (L'Extase matérielle) impliquent une volonté de dénuement, qui prend plusieurs aspects. D'abord sur le plan formel, il adopte un style simple qui n'évite pas les "il y a", les présentatifs "c'est". Mais la gamme reste étendue entre le style chatoyant et le cri de Mydriase. Sur le plan narratif, "l'événement" peut être minimal au sein du texte bref ainsi de "La Montagne du dieu vivant": une seule action, un seul lieu, un seul personnage Jon. Le récit se résume facilement: Jon gravit une montagne, cette ascension dans le vent devient initiation et opère une forme d'extase, d'endormissement à la suite duquel il voit un enfant divin, il contemple le cosmos puis l'enfant disparaît et Jon rentre chez lui. Au plan thématique, le minimal prend la forme d'un micromonde qui fusionne avec le macromonde dans un rapport d'invariance d'échelle, qui est le propre des fractales ${ }^{17}$. Un des propos tenus par Le Clézio au sujet de sa "façon d'écrire" peut corroborer cette hypothèse: "Je me souviens que j'avais appelé ma façon de travailler la technique du chou-fleur. Tout était au centre et le roman autour" ${ }^{\text {18. }}$.

Peut-on parler d'esthétique du détail (comme l'atteste le punctum des photos prises par l'auteur dans La Guerre)? N'est-ce pas en contradiction avec l'esthétique de l'horizon et des lignes de fuite ou de sens pour lesquelles la critique leclézienne affirme ses préférences?

Notre typologie de trois minimalismes - formel, narratif et thématique - qui permet de cerner ce dont on parle n'a aucun intérêt si ne préside pas la question essentielle: pourquoi cette

\footnotetext{
${ }^{16}$ Bruno Thibault, dans la pensée de Blanchot, pense à une écriture du désastre: dans Trois villes saintes, "marche de l'écrivain", "Le Clézio met en scène un espace de la disparition et de la désolation, un espace de la catastrophe qui apparaît à la fois précolombien et postmoderne“, Le Clézio ou la métaphore exotique, Rodopi, 2009 , p. 62.

${ }_{17}$ Claude Cavallero y fait une seule allusion fort pertinente mais sans développer dans Le Clézio témoin du monde (sur la couverture duquel figure une fractale), "la fiction tisse au fil des premiers textes son propre jeu de reprises et de renvois internes de façon singulière - on pourrait presque dire d'une façon qui évoque la dimension fractale“, Calliopées, 2008, p. 151. Roxana Guliciuc-Dreve travaille depuis 2005 à une thèse intitulée "J.-M.G. Le Clézio et Göran Tunström. Analyse fractale du thème de l'enfance“.

${ }^{18}$ Entretien de Guillaume Chérel et de JMG Le Clézio, "Voyageur immobile", Regards, 1er juillet 1997, pp. 1-2. Du moins évoque-t-il son attirance dans les années 60-70 pour la construction mais en lui donnant un sens musical.
} 
option? La réponse est à chercher dans la dimension éthique. Ce ne sont pas les systèmes inventés par les hommes qui donnent sens. Le dépouillement des démunis (exclusion sociale et rédemption de ceux qui vivent en marge du consumérisme), des déshérités (mythe personnel) engage à voir le monde autrement que dans un dualisme, entre deux échelles par exemple, mais à penser la continuité ou l'espace tiers de flottement, de dessaisissement des certitudes. D'où une détotalisation du monde en éclats et poussières, une fréquence des grains de sable et autres métaphores de la décomposition pour un écrivain qui écrit que "l'artiste est celui qui nous montre du doigt une parcelle du monde..." (L'Extase matérielle). Ces épisodes participent-ils de la refabulation du monde, du retissage dans un espace littéraire si travaillée par la dégradation, et analysée promptement comme déconstruction postmoderne, chute des grands récits, principe de l'éclatement au risque d'occulter alors l'éclat, sa lumière? Le minimalisme qui engage cet autre regard sur les éclats est la voie prise par Georges Lemoine, l'illustrateur de Mondo. Loin des déclinaisons géographiques que proposent les deux documentaires filmiques sur l'auteur, les créations de Lemoine visitent l'intime, ses propres obsessions en les déterritorialisant, en majorant incontestablement les choix minimalistes. Le régime du mineur, du délaissé, du "presque rien" selon l'expression de Jankélévitch lui est propre ${ }^{19}$. Lors de sa première rencontre avec Le Clézio, Georges Lemoine lui avait dit "que la lecture de ses romans avait changé quelque chose dans [s]a pratique du dessin; celui-ci devenant plus incisif, plus précis, plus minimaliste... les petites choses représentées, plumes, pierres, sables... prenant sous [s]es doigts tout leur sens, trouvant véritablement leur place dans l'univers". Le courrier que Le Clézio lui adresse en 1988 fait à son tour de l'illustrateur un conteur: “... Quelle belle histoire que celle de vos dessins, qui parle d'herbes et d'arbres, de pierres et de sable, de fumées, de nuages, de reflets dans les yeux des jeunes filles et des rides au coin des yeux des vieux sages“. Dans le recueil Mondo et autres histoires, réédité en avril 2009 chez Gallimard Jeunesse, G. Lemoine dessine pour "La Montagne du Dieu vivant" une pierre à l'image de la montagne le définissant ainsi comme objet fractal par une invariance d'échelle. Quel que soit le point d'où on l'envisage, son irrégularité est identique. Microcosme métonymique, invariant, "le caillou avait exactement la forme de la montagne" dit le texte. La pierre, dans le récit, métamorphose en hiérophanie (quelque chose de sacré qui se montre à nous), autant dire en un archétype collectif. Ce bloc de lave bénéficie des attributs de son volcan originel, symbole de l'énergie première du cosmos. Le corps de Jon, l'enfant qui gravit la montagne, trouve son décor, l'infiniment petit se raccorde avec l'infiniment grand, d'une manière peutêtre panthéistique, symbiotique, chamanique (puisque les textes présentés ici sont contemporains ou postérieurs à la période d'initiation de Le Clézio chez les Emberas), ce qui

\footnotetext{
${ }^{19}$ Voir le livre/catalogue Georges Lemoine, écrire-dessiner, Scriptorial, Avranches, 2008. Notamment le texte d'Elizabeth Brami.
} 
a forgé une œuvre métisse (La Pensée métisse, Gruzinski), mêlée d'héritages français (phénoménologie), amérindien (chamanisme) et mauricien (culture de l'oralité, du conte et des énigmes).

Si Le Clézio a tracé son chemin hors des grands courants littéraires de son époque y compris le Nouveau Roman, il s'est toutefois imprégné de l'accent mis par Robbe-Grillet sur la dimension extraordinaire du détail, sur l'être-là du monde. "Le monde n'est ni signifiant, ni absurde. I/ est tout simplement."20 Barthes explique comment le roman de Robbe-Grillet "est terrestre; il enseigne à regarder le monde [...] avec les yeux d'un homme qui marche dans la ville sans autre horizon que le spectacle, sans d'autre pouvoir que celui-là même de ses yeux" ${ }^{\prime 21}$. Ce propos correspond à bien des pages lecléziennes, ainsi d'un extrait des Géants où Bogo le muet regarde des galets. La lumière "dure" du soleil "cherche à crever les yeux" est-il répété. Elle s'annonce comme porteuse d'événement, elle illumine "toutes les parcelles de mica". Et Bogo en est alors pénétré: "Il voyait tout, et cela entrait en lui en roulant des tourbillons de feu, des étoiles, des spirales, des trombes d'étincelles dans le ciel noir" (G, 103). Cependant la plage est divisée en deux: d'une part, l'espace de la brûlure avec les gens qui crient, "I'horizon" et le "soleil" paralysant qu'évite de regarder Bogo et, d'autre part, la plage de galets vivants, servant de points de repères, montés en pyramide comme chez les Indiens Navaho ou les Chédis de Birmanie ou disposés en cercle magique. Si l'on fait abstraction de l'allusion initiale à la racine/souche de la nausée sartrienne, la relation avec le galet oriente la lecture vers le geste rituel vain puisque Bogo qui aurait voulu devenir galet n'y parvient pas et a peur. Le passage de l'autre côté ne s'effectue pas. À la fin du récit, un incendie révolutionnaire défie cette ville à l'œil panoptique et à l'électricité rayonnante où le personnage de Bogo ne peut parler et doit "plisser les yeux". Le Clézio fait sentir la vibration de la plage mais Hyperpolis où se déroule Les Géants est une anti-utopie où le désir est sous contrôle. II en va tout autrement dans la nouvelle "La Montagne du dieu vivant", centré sur la correspondance du petit et du grand, et sur un seul événement ou chemin vers l'extase matérielle. Un autre dessin de Georges Lemoine, que celui mentionné plus haut, est celui de Jon arrivé au sommet de la montagne qui accompagné d'un enfant divin apparu contemplant la lumière d'une spirale. La spirale, motif chamanique, fait retour sur elle-même en échappant à la fermeture du cercle, à la répétition de l'Histoire, elle sied à notre monde brisé. Elle est la figure emblématique de la théorie du chaos qui s'intéresse au réel, aux nuages... La pierre de Jon est bien un objet fractal, selon la définition de Mandelbrot, car elle a la même forme et structure en dépit du changement d'échelle. Le roman puzzle de Le Clézio peut aussi être qualifié de fractal (fragment à l'échelle de la phrase, de la page qui converge vers une schize globale cohérente dans ses niveaux) soumis au désir d'Adam

\footnotetext{
20 "Une voie pour le roman“, Pour le nouveau roman, éditions de Minuit, Paris, 1961, p. 18.

${ }^{21}$ Roland Barthes, "Littérature objective", in CEuvres complètes.
} 
Pollo. Ce désir, selon Deleuze et Guattari, "est révolutionnaire parce qu'il veut toujours plus de connexions et d'agencements", ces rhizomes qui éloignent de la racine au sens deleuzien.

\section{Quelle lumière?}

Trois Villes Saintes est un mouvement, une marche sans horizon, Mydriase commence par une nuit noire, "sans luciole", "La Montagne du dieu vivant" offre un instant épiphanique, une parenthèse d'éternité, comme par effraction, parce que le récit se tisse dans une temporalité suspendue et se lit à la lumière des archétypes collectifs. L'épiphanie qui traverse diverses religions comme l'a démontré Mircéa Eliade, est une lumière crue, une apparition comme dans le modèle judéochrétien. Les visages dans l'univers romanesque de Le Clézio sont des visages-paysages épiphaniques au prisme de cultures diverses: ils engagent à la responsabilité comme ceux de Lévinas, ils sont aussi similaires aux gros plans cinématographiques qui ont fasciné Le Clézio dans Ballaciner, aux visages photographiés dans L'Africain, aux visages peints de Modigliani décrits par l'écrivain...

Notre corpus réunit des textes brefs qui demandent un lector in fabula ${ }^{22}$ plus disposé à fournir un effort de co-création du sens. Dès lors de multiples questions se posent: s'agit-il de retrouver l'Un dans le Tout? D'une vision fractale? D'une annulation éthique des hiérarchies? D'une phénoménologie à l'œuvre? De vivre à l'échelle de l'infiniment humain sans ambition leurrante d'embrasser une totalité? D'annuler la dichotomotie micro (détail) et macro alors qu'elle structure indéniablement le texte leclézien? Et, entre le petit et le grand, qu'opère l'espace du jeu, d'entre-deux? Une stimulation de la myopie? Un espace de confusion? Un métissage des formes? La critique a vite recouvert la question soit par la classification des détails et du fragment comme signe d'œuvres de rupture typique de la modernité, du désenchantement ${ }^{23}$, soit par la célébration vibrante mystique de l'extase. Les deux pistes interprétatives figurent d'ailleurs deux doxas: la première plus en relation avec l'approche sociohistorique de l'œuvre, la seconde avec l'approche mythique. Toutes deux pertinentes ces analyses ont cependant l'inconvénient de se tourner le dos. Car la singularité de l'œuvre est précisément d'être appréhendable par les deux. L'opération intellectuelle qui consiste à analyser séparément ne rend pas compte du fait que les deux agissent le texte en même temps et en font un lieu d'incertitude et de paradoxe. Un lieu déconstruit (ce que la microanalyse pointe) et un lieu de fabulation qui au fil des ans génère une poétique du lien en dépit du désenchantement, un lieu de déconstruction qui n'est pas englouti dans la

\footnotetext{
${ }^{22}$ Comme le rappelle Michel Viegnes "Événement et temporalité dans les récits brefs de Mondo et autres histoires", Cahiers JMG Le Clézio numéro 2, op. cit. 2009.

${ }^{23}$ Voir l'analyse rigoureuse d'Isa Van Acker, Carnets de doute, Rodopi, 2008.
} 
terreur de l'histoire contemporaine. Entre disparition (réduction en poudre, civilisation disparue) et apparition, ce qu'est la trace, l'empreinte et la survivance. Entre vie et survie, une revivance à laquelle conduisent les femmes dans les récits récents.

Pour finir, nous nous en tiendrons à une allusion à L'Enfant de sous le pont. Dans un dispositif énonciatif oral, l'incipit de la nouvelle-conte pose un décor du dénuement, caractéristique de la pauvreté des immigrés, mal intégrés. L'histoire est celle d'un SDF, estrassier (ramasseur de chiffons), ancien Harki, qui trouve un bébé abandonné déposé, sous son pont. II le nourrit, puis trop pauvre finit par le donner à une famille. Á plusieurs niveaux Le Clézio joue avec le modèle générique du conte et du roman familial, où les orphelins sont rarement adoptés. La force du regard de l'enfant, comparé à des bijoux, dont une description précise fait défaut est pointée au passage sans aucun éclairage forcé. La rencontre du SDF et du bébé finit par une séparation certes, mais aussi par une revivance, très éloignée de la noirceur des nouvelles inspirées de fait divers contemporains, par le don de l'enfant à une famille d'adoption et dans la façon de rendre actif et bienveillant le SDF. L'Enfant de sous le pont qui commence par un abandon est une histoire de don, de passation, et donne ce qu'on appelle une lueur d'espoir.

L'engagement dans une forme de dénuement dans la tradition des opprimés et l'affirmation progressive de l'autobiographie orientent l'œuvre actuelle de Le Clézio vers des écrits de restitution (voire de réparation avec L'Africain). Ce que corroborent les dons d'objet, autres formes de transmission à l'œuvre dans les récits. Le désenchantement des premières œuvres ou la défaite des utopies dans le récent Ourania (qui est aussi une scrutation des étoiles remise en question) résulte d'une vision élargie du monde d'un écrivain passant, tel Benjamin, qui offre certes parfois un ange de l'histoire aux yeux écarquillés mais aussi un bébé au regard de bijoux. Notons que la naissance, événement de Mydriase, est un thème récurrent et de clôture de cinq de ses romans.

\section{Savoir nuage}

L'œuvre de rupture s'inscrit dans une génération de désenchantés mais c'est aussi une œuvre de l'ouverture, de la liaison entre les cultures, posant des actes de restitution ou de legs. L'effet de projecteur sur "le minimalisme de l'esprit" ${ }^{24}$ comme dénuement ne relève pas d'une démarche essentialiste mais d'une pensée plus primitive, qui fait du désert, d'un galet, d'un bloc de lave, d'un chemin un espace sans fin et lisse. Car en parcourant ces quelques récits dans l'ordre de parution, tout en pointant l'évolution de l'œuvre de

\footnotetext{
${ }^{24}$ Expression de Le Clézio dans G. de Cortanze, J.M.G. Le Clézio, le nomade immobile, éditions du Chêne, Hachette livre, 1999. Entretiens de novembre 1997 à janvier 1999, édition Folio n³664, p. 89.
} 
l'éclatement vers l'éclat, nous insistons sur les relations entre les échelles et sur les entredeux, véritables opérateurs de structures. Entre la nuit et le jour, entre le cri et le silence, entre l'immobilité et le mouvement, il est un espace étoilé, un espaces de mots, un mouvement de spirale.

Avec Le Clézio, le temps des nuages se substitue à celui des horloges, pour reprendre une distinction de Poper. L'horloge c'est la précision, largement enrayée par l'absence de données temporelles, par l'itératif et le chronotope qui ralentissent le récit. Le nuage c'est l'indécis, l'instabilité et le mouvement. L'exergue de Ballaciner ne nous invite-t-il pas à "tomber du ciel de nuage en nuage", à nuager et combien de fois L'Inconnu sur la terre n'est-il pas aux nuages?

Selon Serge Gruzinski, "les métissages relèvent de cet ordre de réalité". Son "modèle du nuage" suppose "que toute réalité comporte une part de méconnaissable et qu'elle recèle toujours une dose d'incertitude et d'aléatoire" ${ }^{25}$. L'aléatoire participe des systèmes les plus élaborés aux simples "grains de poussière" et l'incertitude participe non de l'effondrement mais de la fragilité des entre-deux, de la quête des lueurs, celle du Chercheur d'or d'abord séduit par les histoires de trésor de corsaire et insensible aux liens de la terre et du ciel qui comprend en définitive, grâce à la métisse Ouma, les voies de la dépossession, celles de la montreuse d'or Iwa. La quête des lueurs et des étoiles dessine alors un espace politique de contre-pouvoir, ou comme l'exprime Didi-Huberman (2009), à propos du cinéma de Pasolini, qui n'annonce pas une apocalypse finale (et la fascination pour la disparition) mais des lumières passagères, des images passantes, des "ouvertures, des possibles, des malgré tout".

\footnotetext{
${ }^{25}$ Serge Gruzinski, La Pensée métisse Paris, Fayard, 1999, pp. 53 à 55.
} 


\section{Bibliographie}

Collectif dir. Yan Ciret (1997). Archipel Lavaudant. Christian Bourgeois.

Collectif Revue dir. Bruno Thibault et Claude Cavallero (2009). Cahiers JMG Le Clézio n2, Contes, nouvelles, et romances. Paris: Complicités.

Cavallero, Claude (2009), Le Clézio, notre témoin. Calliopées.

De Cortanze, Gérard (1999). J.M.G. Le Clézio, le nomade immobile. Paris: éditions du Chêne.

DEWULF, Bernard (2009). Peintures et scénarios. Robert Devrient, Musée des beaux arts de Tourcoing.

DIDI-HuBERMAN, Georges (2009). Survivance des lucioles. Paris: Minuit.

DuPONT-MONOD, C., "Un lieu d'incertitude", entretien avec Le Clézio, Page des librairies, novembre 2000.

FouCAULt, Michel (1946). "Le langage de l'espace". Critique. Paris: Minuit.

GRUZINSKI, Serge (1999). La Pensée métisse. Paris: Fayard.

LE CLEZIO, Jean-Marie Gustave (1973). Les Géants. Paris: Gallimard, pp. 101-109.

LE CLEZIO, Jean-Marie Gustave (1973). Mydriase. Paris: Fata Morgana.

LE CLEZIO, Jean-Marie Gustave (1973). "Le Génie du Datura". Cahiers du chemin. NRF n917, janvier 1973, pp. 95-129.

Le ClezIO, Jean-Marie Gustave (1978). "Peuple du ciel" et "La Montagne du dieu vivant", in: Mondo et autres histoires. Paris: Gallimard.

LE CLEZIO, Jean-Marie Gustave (1980). Trois villes saintes. Paris: NRF Gallimard.

LE CLEZIO, Jean-Marie Gustave (2001). L'Enfant de sous le pont. Lire c'est partir.

LE CLEZIO, Jean-Marie Gustave (2004). L'Africain. Paris: Mercure de France.

LE CLEZIO, Jean-Marie Gustave (2007) Ballaciner. Paris: Gallimard.

Robbe-Grillet, Alain (1961), “Une voie pour le roman”, Pour le nouveau roman. Paris: Minuit.

SALLES, Marina (2006), Le Clézio, notre contemporain, Rennes, PUR.

THIBAULt, Bruno (2009), Le Clézio ou la métaphore exotique, Amsterdam: Rodopi.

VAN ACKER, Isa (2008), Carnets de doute. Amsterdam: Rodopi.

VIEGNES, Michel (2009), "Événement et temporalité dans les récits brefs de Mondo et autres histoires", in: Cahiers JMG Le Clézio n². Paris : Complicités. 